\title{
Brain Computer Interfaces, a review
}

\author{
by Shirley Coyle, Tomás Ward and Charles Markham
}

"Men ought to know that from the brain, and from the brain only, arise our pleasures, joy, laughter and jests, as well as our sorrows, pains, griefs and tears. Through it, in particular, we think, see and hear, and distinguish the ugly from the beautiful, the bad from the good, the pleasant from the unpleasant." Hippocrates, The Sacred Disease XVII [1]

The brain is in many respects the centre of our being, controlling our actions, movements, thoughts and emotions. It is somewhat of a mystery, presenting itself only through the body's exterior façade. It is safeguarded by a thick skull that insulates it from the outside world. Information from the surroundings is relayed to it via the five senses - touch, sight, sound, smell and taste. Its role was underestimated in the past by cardiocentrists who believed that thought, sensation and behaviour originated in the heart and that the brain was there to "make the heat and boiling in the heart well blent and tempered"-Aristotle(384-322BC). Today we can pinpoint the areas of activity in the brain, and localise its functions. We now have an understanding of the physiological processes and signals that occur. We know that neurons in the brain's cortex transmit signals to an efferent nervous system, i.e. from the brain towards motor output pathways, and also from an afferent system, i.e. from the sense organs to the brain. The impulses are both electrical and chemical signals, which can be detected and measured as with any system using specific techniques. As with any system if there are problems we want to solve them, if there are improvements to be made we implement them where possible. Problems can occur in the afferent system, and also in the efferent system, for example causing visual or auditory impediments in the first case and paralysis in the latter. By effectively "bypassing" the nervous system the brain can be connected in a more direct sense to its environment. Brain computer interfaces offer this possibility. Their origins lie in providing alternative communication methods for the disabled, but now offer the possibility of providing people with "different senses". These augmentative channels will allow the brain to directly connect to its environment. Electrical signals originating from the brain can be directly sent to computers, providing it with an additional control output. This can even be connected to the Internet to provide an "extended nervous system" for controlling a robot hundreds of miles away. Conversely new and different senses such as ultrasound or infrared detection could be relayed as sensory information to the brain. In the exciting information age we live in today, integrating technology with our biological systems seems like a natural progression, and obtaining a "silicon nervous system" may by the only way to keep up!

\section{The Driving Force}

The initial motivation behind brain computer interfaces was to develop communication devices for the severely disabled. In particular for cases where no motor output can be relied on, where pointing, tapping or eye-blinks are not even an option. The majority of us are lucky in that we are able to express ourselves how and when we want - voice our opinions, desires, needs, and essentially interact with the people around us, not only through conventional face-to-face confrontations but also through the many telecommunications media available to us. For people with severe 
illnesses affecting motor functions, logging on to the internet or even holding a simple conversation is a much more arduous task. Amyotrophic Lateral Sclerosis, for example, is a condition that causes the degeneration of motor neurons, resulting in total paralysis and in the later stages patients need artificial ventilation [2]. In the case of "locked-in syndrome", patients are conscious but unable to move their muscles, with the exception of vertical eye-movement and blinking in some cases. Whatever the cause of the motor impairments, advances in medicine enable these patients to survive and live longer, advances in biomedical engineering and computing should now be able to assist in improving the quality of their lives by allowing them to convey their thoughts and desires and communicate with the world.

Brainwaves?

"The brain is a tissue. It is a complicated, intricately woven tissue, like nothing else we know of in the universe, but it is composed of cells, as any tissue is. They are, to be sure, highly specialized cells, but they function according to the laws that govern any other cells. Their electrical and chemical signals can be detected, recorded and interpreted and their chemicals can be identified; the connections that constitute the brain's woven feltwork can be mapped. In short, the brain can be studied, just as the kidney can." David H. Hubel - (1981 Nobel Prize Winner) [3]

One such method of detecting the electrical signals of the brain is the Electroencephalograph or EEG, which was first shown by Dr. Hans Berger in 1929[4]. Using this method electrical activity ("electro") of the brain ("encephalo") can be measured and recorded ("graph") without penetrating the skull. This is done non-invasively by connecting electrodes to the scalp. The detected EEG activity is quite small, with signals measured in microvolts $(\mu \mathrm{V})$ and the main frequencies of interest are up to approximately $30 \mathrm{~Hz}$.

\section{Figure 1:}

Electrical activity detected by EEG forms the basis for many of today's braincomputer interfaces. Various research groups have developed brain-computer interfaces using their own twist to "translate" the brain's electrical signal. There are various methods of processing the signal, some of the current systems use frequency domain features of the signal, others time-domain. The user learns to adapt and control specific brain signals while the machine must also learn to recognise characteristics of that particular user. Adaptability is the key, and the human brain is extremely adaptable - when it recognises benefits within its vicinity it will jump on the opportunity. Learning to control particular waves (rhythms) can be used to provide a new output channel for the brain. EEG signals are often classified in terms of frequency, e.g. delta $(1-4 \mathrm{~Hz})$, theta $(5-7 \mathrm{~Hz})$, alpha $(8-12 \mathrm{~Hz})$, beta $(13-22 \mathrm{~Hz})$. Each waveband, or rhythm is associated with different tasks, e.g. alpha wave are more prominent when the body is in a relaxed state. By learning to control the electrical activity, and constructing systems to understand these signals, an alternative output channel for the brain can be created. This is exactly what a research team, led by Dr. J.R. Wolpaw, in the Wadsworth Centre, New York has done [5]. They have developed a brain computer interface that uses particular waves (rhythms) that occur above the motor cortex, mu and beta waves. With the help of motor imagery users were able to learn to control their mu and beta rhythms to control a cursor in both one and two-dimensional space. 
Other BCIs analyse the power of the EEG rhythms, changes are known as EventRelated Desynchronization and Event-Related Synchronization (ERD/ERS). ERD corresponds to the decrease in power of an EEG rhythm related to an event.

Conversely, ERS corresponds to an increase in amplitude of an EEG rhythm related to the event. Left or right hand movements will cause ERD in the contralateral hemisphere of the brain in the motor cortex. It has been known for some time that the signals produced in preparation for a physical movement are the same as the signals produced during the actual movement. In fact ERD can be detected up to $2 \mathrm{~s}$ before movement. Dr. G. Pfurtscheller's "Graz BCI" has used this method to develop a cursor-control system and has also demonstrated the control of a hand prosthesis by imagination of right and left hand movements [6].

Another method with is more dependent on the normal neural pathways is to use what are called Visual Evoked Potentials (VEP). What this means is that when a particular stimulus occurs, e.g. a flashing light, it can lead to a specific electrical signal, an event-related potential. Several trials must be performed and the results averaged to obtain a result. In terms of implementing a brain-computer interface visual eventrelated potentials have been used by presenting the user with a number of letters/symbols on a screen, and sequentially flashing each item on the screen [7]. The user must focus their gaze on their desired choice, and when this item flashes it activates a VEP, measured at the occipital cortex (area of the brain related to sight). There is a major drawback to this approach however - that is users must be able to control their gaze direction. Ideally a brain-computer interface should be independent of the normal motor or sensory conduits of the brain.

So does a brain-computer interface correlate with mind reading? A "Thought Translation Device" developed by Dr. Neils Birbaumer [8] in the University of Tubingen, Germany, may sound like an overly ambitious title to some. However, seeing as it allows users to compose phrases and sentences just by thinking, it really does live up to its name. This device has enabled patients suffering from amyotrophic lateral sclerosis to use a language support program. The system used takes yet another approach in the analysis of EEG signals - it uses slow cortical potentials that are features of the signal that occur from 300ms up to several seconds/minutes after a stimulus. Using biofeedback patients can learn to control their slow cortical potentials to operate this device.

Communication rates and accuracy are an issue with the current EEG-based braincomputer interfaces, and naturally vary from patient to patient. Research is on-going however, both in terms of processing the signal and also developing the user interface to enable faster control e.g. new word prediction algorithms. The brain can adapt, this is evident from evolution, and with computers at our command we can integrate with their computational efficiency, effectively increasing our own bandwidths.

Digging deeper

The EEG-based BCIs have attractive prospects, but as with everything in life there are always disadvantages. EEG delivers quite a complex signal for processing, which unfurls from the electrical signals of millions of neurons, having passed through the skull before being detected by electrodes on the scalp. What if we could somehow get right down to an individual neuron's level? Obtaining a direct signal from a single neuron could certainly make it easier to "listen in" on the brain's signals. Well it is possible, and it has been done. Obviously to do this does necessitate surgery. 
Electrodes must be implanted into the brain's cerebral cortex in order to achieve a direct interface. The first electrode to be approved by the Food and Drug Administration (FDA) for human use was developed by Neural Signals Inc. In spite of the invasive nature of such implants there has been much success in the past and endless possibilities for the future.

A research team in Atlanta, led by Dr. Philip Kennedy, CEO Neural Signals Inc. [9], have successfully demonstrated a brain computer interface that uses an electrode implanted into the motor cortex of the brain [10]. The implant consists of a hollow glass cone that intercepts neural signals by making a direct connection to the neurons as they fire. Trophic factors are used to encourage the growth of neurons into the cone to make the connection. When a signal is transmitted electrical pulses are detected by two wires, amplified and transmitted to a nearby receiver, where the signals can then be processed, analysed and used to control a cursor. Controlling the cursor allows phrases to selected and then synthesised or alternatively letters can be selected from a visual keyboard in order to communicate. A patient suffering from "locked-in syndrome” is using this BCI successfully. The system not only acts as a spelling device but also allows him to control the air conditioning of the room he resides in. In this way he can interact with his environment through direct coupling to his brain. Dr. Kennedy believes that this technology will lead to "movement restoration, robotic control (if needed) and eventually, (you guessed it, scary thought): Cyborgs" [11].

\section{Figure 2}

Last year scientists from the Duke University Medical Centre, MIT and the State University of New York (SUNY) Health Science Centre used monkeys to control a robotic arm using just their minds [12]. As the monkeys moved their arms their brain signals were detected and deciphered, and used to move a robotic arm mimicking their own movements. As an experiment the signals were transmitted over the Internet to control a robotic arm hundreds of miles away. The Internet was effectively an extension of the monkey's nervous system allowing it to reach his arm across to a distant lab. Almost a hundred electrodes were implanted into the monkey's brain to enable the movement of the arm in three dimensions. Last year, in the Brown University, a similar breakthrough was made. Using the same technology a monkey was trained to control a cursor by thought. The implant differs from the glass cone used previously in Atlanta as it actually consists of an array of electrodes, so signals can be taken from a range of neurons. With the use of various functional brain imaging techniques, and their improvements in their resolution, identification of the specific neurons to be connected will be possible. More recently, researchers in Arizona State University found that by providing feedback to the monkeys they could control a 3D cursor. The monkeys first learned to move a ball around a 3D space on a computer screen first by hand movements and then just by thought [13]. In the future, connecting numerous electrodes to various neurons should allow enough precision for use in control of prosthetic limbs and possibly restoring muscular control to paralysed patients.

Biological signalling network down? Computers to our aid.

The possibility of controlling a machine with our minds lends the question as to whether or not the inverse scenario is possible, i.e. if we can detect electrical signals from the brain and understand them, in so far as is possible, can we then artificially produce these signals for the brain? It has been shown that stimulating exposed brain 
tissue with electric current triggers the corresponding function of the stimulated area. A German doctor, Eduard Hitzig, who worked in a military hospital in the 1860's conducted some experiments on soldiers whose brains were partially exposed as a result of war injuries. He stimulated the exposed cortex using wires connected to a battery and found that weak electric shocks applied to the back of the brain caused the patient's eyes to move. He later conducted experiments on dogs, and was able to crudely control their movement. Over the coming years scientists commonly used animals for such experimentation, and almost a century later a neurosurgeon called Wilder Penfield was able to map the motor cortex of the brain. While operating on a patient he used a mild electric current to stimulate areas of the motor cortex and observe the resulting movements. He also probed areas of the temporal lobe; an area associated with memory, and found that the patient, who was conscious throughout surgery, recalled various memories vividly upon stimulation. The principles demonstrated by such scientists have led to development and research into applications in medical care, for rehabilitation and therapy.

Epilepsy is quite a common condition affecting about 1 in 200 people, caused by abnormal electrical activity of the neurons in the brain. Epileptic seizures prevent the brain from interpreting and processing incoming sensory signals and controlling muscles. Intercepting these abnormal neural firing signals can prevent seizures. A system commonly referred to as a "pacemaker for the brain" offers an alternative to medication and surgery for those suffering from epilepsy. This device is a NeuroCybernetic Prosthesis System (NCP System), marketed by Cyberonics, Inc. [14] The system consists of a pulse generator and electrode that emits electrical signals to the brain to interrupt the signals that occurs before a seizure. A programmable chip controls the pulse generator that is implanted in the patient's chest, and tunnelled under the skin to the neck near the vagus nerve. Intermittent signals are sent, but the option is there for the patients to trigger the device themselves at the onset of a seizure, simply by swiping a magnet across their chest above the implant. The first implant of a vagus nerve stimulation device was back in November 1988, and since then possibilities of using this technology for the treatment of other conditions, such as depression, have been investigated with promising results.

A neurostimulator has been developed to help control the symptoms of Parkinson's disease. This disease affects movement with symptoms including tremors, rigidity, bradykinesia/akinesia(slowness/absence of movement), and postural instability. These symptoms can prohibit patients from performing their daily activities. A therapy developed by Medtronic Inc. uses an implanted medical device to deliver electrical stimulation to block the brain signals that cause symptoms of Parkinson's disease [15]. The treatment is known as Activa Parkinson's Control Therapy and involves implanting a neurostimulator beneath the skin in the chest which is joined via an extension threaded under the skin to the "lead", an insulated coiled wire which is implanted in the subthalamic nucleus or globus pallidus interna of the brain. The neurostimulator generates electrical pulses that are then delivered to these target areas, blocking the signals that cause the symptoms of Parkinson's disease.

\section{Figure 3}

The capabilities of such neurostimulators provokes the question, "how far can we take control of the brain using technology?" Scientists in New York have recently "hijacked" a rat's brain! They effectively turned the rat into a robot, controlling its 
movement. This was done by implanting three electrodes, one in the medial forebrain bundle, an area associated with pleasure, and two in the somatosensory cortex, more specifically in the areas associated with sensations of touch in the right and left whiskers. The rats were trained to move left and right or forward depending on which part of the brain was stimulated. Because of their size and agility these creatures, nicknamed "roborats", are ideal candidates for searching through rubble for survivors in cases of building collapses. The rats can be sent in with video-cameras fastened onto their backs, and steered remotely.

The thought of being able to turn rats into robots may install terror in the minds of many, fearful that this technology may get into the wrong hands, and wonder what the world is coming to. Is this evolution a bad thing? Undoubtedly they have a valid point, and the ethics of taking total control of humans in such an extreme way has to be considered. Having said that, the research into this field that inevitably helps with rehabilitation and improving the quality of people's lives has definitely to be admired. Computer vision has been given a whole new dimension as computers have been used to see the world through the eyes of one of the rats' predators - the cat. Researchers in the Department of Molecular and Cell in the University of California connected a computer to a cat's brain and recorded videos of what it was visualising [16]. Optic nerve fibres from the eyes terminate at two sections in the thalamus (a structure in the middle of the brain) known as the Lateral Geniculate Nuclei LGN, one in each hemisphere. By connecting electrodes to cells in this area they "reconstructed natural scenes with recognizable moving objects" by using decoding algorithms. They were effectively given a cat's eye view of the world, and even able to distinguish people's faces. Through a better understanding of the sensory systems, the original sciencefiction type ideas of human-machine interfaces through connections with the central nervous system are beginning to become fact.

Restoring sight to the blind may sound like the job of a miracle worker, but now there are commercial systems available to do just that. The Dobelle Group in New York, after 30 years research, has made artificial vision a reality $[17,18]$. The "eyes" of their system consist of a miniature camera and an ultrasonic distance sensor, mounted on a pair of eyeglasses. The information these observers capture is dispatched via the "nerves" of the system to a computer, where the signals are processed, which is compact enough to be attached to a person's belt. Once necessary algorithms to simplify the image and eliminate noise have been applied the computer then triggers a second microcomputer that transmits pulses to an array of 68 platinum electrodes implanted on the surface of the brain's visual cortex. Stimulating the visual cortex in this way has given a man enough vision to drive a car.

\section{Figure 4}

\section{Magnetic Effects}

When Faraday described the induction effects of a changing current in a coil of wire to the Royal Institution of Great Britain in 1831 who would have though that the human brain could be used as the second coil of wire? At the end of the 19th century a French physicist and physician Jacques-Arsène D'Arsonval showed the effects of magnetic fields on the body [19]. He was a pioneer in electrotherapy, and the terms "darsonvalisation" and "diathermy", still used in physiotherapy today, stem from his research. He found that by surrounding the head with a time-varying magnetic field caused one to see flashes of light. This is due to an electric current induced on the 
retina. In 1985 the first stimulation of the motor cortex, using magnetic fields, was performed by Barker et al [20]. This was the beginning of Transcranial Magnetic Stimulation (TMS)[21].

\section{Figure 5}

Since then the cortical effects of TMS have been observed directly, by means of various brain imaging techniques such as electroencephalography (EEG), positron emission tomography (PET) and functional magnetic resonance imaging (fMRI). These imaging techniques also allow specific areas of the cortex to be targeted for stimulation. By placing coils of wire carrying pulses of current close to the scalp a changing magnetic field is created around the head. This in turn induces current to flow in the neural tissue of the brain. For example, if part of the motor cortex related to the thumb is stimulated, a current in induced in this cortex and a signal is sent via the central nervous system to the thumb causing it to twitch. The upshot of this is that it enables the examination of motor pathways, and allows the efficiency of the transmission of signals along these pathways to be investigated.

Not only can TMS examine pathways between the central nervous system to the periphery system, with TMS it is possible to examine cortical excitability as well. It is for this reason that pharmacological studies have also found uses for this technology. By measuring cortical excitability and also the transmission of signals within the brain itself between various functional regions, the effects of various drugs can be examined. Studies have been carried out on the effects of epileptic drugs [22], and also the effects of alcohol on the brain. While testing patients suffering from Parkinson's disease using TMS therapy, it was found that those who felt depressed actually experienced a feeling of well-being after the therapy [23]. This triggered possibilities of using TMS to treat neuropsychiatric conditions such as depression [24]. In those suffering from clinical depression there appear to be areas of the brain which have lower neuronal activity, in particular the left prefrontal cortex. This is an area that is easily accessible for TMS therapy, and increasing the neural signals in this area using TMS is being increasingly used to combat depression.

Not only can TMS be used to stimulate movement and cause neurons in the brain to fire, it can also be used to disturb on-going neuronal signals. This is important in the quest for neuroscience to truly understand the brain, allowing the identification of cortical areas that are important for specific tasks. By generating and also preventing neural signals we can actually use machines to provide direct control for the human brain where necessary.

What next?

So what does the future hold for brain-computer symbiosis? Many believe that humans and computers will be integrated as one. The technology is there, (well almost), and the urge to live the reverie of science fiction is ablaze. "Scary? Cyborgs are inevitable" says Dr. Phil Kennedy, CEO Neural Signals Inc [11]. These are of course questions regarding the morality of enhancing humans by using electronic and computer technology. Medicine and its associated Sciences have instinctively always tried to fix things, but there is always the question of taking things too far. Where does one draw the line as to what is superfluous? In the future surgery for electronic implants for new and different senses may become as commonplace as plastic surgery 
is used today to improve the body's exterior image? Maybe it's time to let our brain that remains hidden from the scene have its turn to enhance its current means by availing of new input and output channels. Professor Kevin Warwick, the worlds first Cyborg, has extended the limitations of the human body by showing that it is possible to upgrade the human system by connecting to a machine. The term Cyborg comes from Cybernetic Organism, an organism integrating the control systems of both the human nervous system and brain, and an electronic/mechanical control and communication system. Prof. Warwick believes that "humans will become Cyborgs and no longer be stand-alone entities". Will this be a "natural" evolution? Stephen Hawkings has been reported to say, "If humans don't watch out, computers will take over the Earth" [25]. In some senses computers are miles ahead of us, their processing speed, computational efficiency and memory outweighs our own. However I truly believe that they won't and can't take over the world on their own. We create and program them, control their learning and they are certainly not our enemy. Human computer communiqué can deliver to us the best of both worlds. Learning is a twoway process, and adaptability must come from both sides of the interface - the human brain, and the computer.

Hippocrates wrote "I hold that the brain is the most powerful organ of the human body...eyes, ears, tongue, hands and feet act in accordance with the discernment of the brain" [1]. Our brains are powerful control centres. They are incisive enough to adapt to their advantage. They make use of the input and output channels available to them as best they can, but these resources have limitations, and can be severed. Our knowledge is continuously growing, and our insight into the human's mechanisms has come a long way since Hippocrates' time. Using our improved understanding we can now provide augmentative channels for our brains' control allowing us to adapt to a changing environment. We can extend the boundaries imposed by our own biological systems and allow our brains to accommodate new senses. These new senses are merely another step in the evolution of man.

\section{References}

1. Martin, G., (1998) Human Neuropsychology, Hemel Hempstead, Prentice Hall Europe.

2. Medline Plus, Health Information http://www.nlm.nih.gov/medlineplus/amyotrophiclateralsclerosis.html

3. Hubel D., (1979) The Brain, Scientific American, Vol. 241, No. 3, pp. 45-53

4. Berger, H. (1930). On the Electroencephalogram of Man. Journal fur Psychologie und Neurologie, Vol. 40, pp. 60-179.

5. Wolpaw,J. R., McFarland,D. J., Vaughan, T. M., (2000) Brain-Computer Interface Research at the Wadsworth Center IEEE Transactions on Rehabilitation Engineering Vol 8, No. 2, June 2000

6. Kalcher, J., Flotzinger, D., Pfurtscheller, G., (1993) Graz brain-computer interface: an EEG-based cursor control system, Proceedings of the 15th Annual International 
Conference of the IEEE Engineering in Medicine and Biology Society, pp. 1264 1265.

7. Middendorf, M, McMillan, G., Calhoun, G., Jones K.S., (2000) Brain-Computer Interfaces Based on the Steady-State Visual-Evoked Response, IEEE Transactions on Rehabilitation Engineering, Vol. 8, No. 2, pp. 211-214.

8. Birbaumer, N., Kübler, A., Ghanayim, N., Hinterberger, T., Perelmouter, J., Kaiser, J., Iversen, I., Kotchoubey, B., Neumann, N., Flor, H., (2000) The Thought Translation Device (TTD) for Completely Paralyzed Patients, IEEE Transactions on Rehabilitation Engineering, Vol 8, No. 2, pp. 190-193.

9. Neural Signals Inc., http://www.neuralsignals.com

10. Kennedy, P.R., Bakay, R.A.E., Moore, M.M., Adams,K., Goldwaithe, J., (2000) Direct Control of a Computer from the Human Central Nervous System, IEEE Transactions on Rehabilitation Engineering, Vol. 8, No. 2, pp. 198-202.

11. Kennedy, P., Personal correspondence

12. Thomson, E.A., (2000) Monkey controls robotic arm using brain signals sent over Internet, December 6,2000, TechTalk, MIT News Office, http://web.mit.edu/newsoffice/tt/2000/dec06/monkeys.html

13. Taylor D.M. , Tillery, S.I., Schwartz, A., (2002) Direct Cortical Control of 3-D Neuroprosthetic Devices, Science, June 7, Vol. 296, pp. 1829-1832.

14. Cyberonics, http://www.cyberonics.com/index.htm

15. Medtronic, http://www.medtronic.com

16. Whitehouse, D., (1999) Computer uses cat's brain to see, Sci/Tech, Friday, October 8, http://news.bbc.co.uk/1/hi/sci/tech/468857.stm.

17. Dobelle Group, http://www.dobelle.com/vision/index.html

18. Kotler, S., (2002) Vision Quest, Wired Magazine, Issue 10.09, September, http://www.wired.com/wired/archive/10.09/vision.html

19. A Biography of Jacques-Arsene d'Arsonval, http://chem.ch.huji.ac.il/ eugeniik/history/arsonval.html

20. Barker A.T., Jalinous R., Frecston I.L., (1985): Non-invasive magnetic stimulation of human motor cortex (letter), The Lancet, 1:1106-1107.

21. Transcranial Magnetic Stimulation - http://www.biomag.helsinki.fi/tms/

22. Jennum P. and Winkel H. (1994). Transcranial Magnetic Stimulation. Its role in the evaluation of patients with partial epilepsy. Acta. Neurol. Scand. Suppl. Vol 152, pp. 93-96. 
23. Ridding MC, Inzelberg R, Rothwell JC. (1995). Changes in excitability of motor cortical circuitry in patients with Parkinson's disease. Ann Neurol Vol. 37, pp. 181188.

24. Pascual-Leone A, Rubio B, Pallardo, Catalá D. (1996). Rapid-rate transcranial magnetic stimulation of the left dorsolateral prefrontal cortex in drug-resistant depression. The Lancet, Vol. 348, pp. 233-237.

25. Perera R., (2001). Hawking: Re-engineer humans or risk machine rule, Cable News Network,

http://www.cnn.com/2001/TECH/ptech/09/05/humans.machine.idg/index.html

\section{Biography}

Shirley Coyle

Shirley studied electronic engineering in Dublin City University, and received her B. Eng in 2000. She then worked with Siemens Ltd. for two years as a project engineer in mobile telecommunications. Her main interests have always been in Biomedical Engineering and she is currently studying for a $\mathrm{PhD}$, in NUI Maynooth, based on near-infrared imaging of brain function.

Dr. Tomás Ward

Dr. Ward received the B.E., M.Eng.Sc. and Ph.D degrees from University College, Dublin, Ireland in 1994, 1996 and 1999 respectively. From 1999-2000 he taught at the Department of Computer Science at the National University of Ireland (NUI), Maynooth. Currently he teaches in the Department of Electronic Engineering at NUI Maynooth. His research interests lie in the areas of human-computer interaction and physiological measurement.

Dr. Charles Markham

Dr. Markham works as lecturer in Computer Science at the National University of Ireland Maynooth. He currently teaches courses in Robotics and Embedded Control. His research interests are in the area of medical imaging, imaging sensors and mobile vision. 